Revue internationale de l'économie sociale

Recma

Pour un lendemain qui ne déchante pas

For a brighter tomorrow

Jean-François Draperi

Numéro 321, juillet 2011

URI : https://id.erudit.org/iderudit/1020856ar

DOI : https://doi.org/10.7202/1020856ar

Aller au sommaire du numéro

Éditeur(s)

Association Recma

ISSN

1626-1682 (imprimé)

2261-2599 (numérique)

Découvrir la revue

Citer ce document

Draperi, J.-F. (2011). Pour un lendemain qui ne déchante pas. Revue

internationale de l'économie sociale, (321), 4-5.

https://doi.org/10.7202/1020856ar 


\section{POUR UN LENDEMAIN QUI NE DÉCHANTE PAS}

A n'en pas douter un nouvel esprit saisit l'économie sociale et solidaire. Les Etats généraux ont vu s'exprimer des penseurs et acteurs nouveaux dont les discours ont mis en avant une nouvelle dynamique et souligné l'importance que la société dans son ensemble prenne la mesure de l'économie sociale et solidaire. Mais de quelle ESS parle-t-on?

Un rapide constat au préalable: si les débats et rencontres ont permis de belles découvertes et de fertiles rencontres, le palais Brongniart a souvent semblé bien vaste. Au-delà du fait que l'assemblée était presque exclusivement francilienne, une large part de l'économie sociale et solidaire n'était ni présente ni représentée aux rencontres. En particulier, les grands acteurs historiques, ceux qui font peser l'ESS dans l'économie - les coopératives bancaires, les coopératives agricoles, les mutuelles de santé, les grandes fédérations d'associations des actions sanitaire, sociale ou sportive - n'étaient pas de la partie, soit parce qu'ils n’ont pas été invités, soit qu'ils n'ont pas souhaité être présents.

Précisément plusieurs leaders ayant organisé les Etats généraux posent la question de l'appartenance de grands groupes coopératifs ou mutualistes à l'ESS. Plusieurs auteurs ont souligné des processus de banalisation des entreprises (Jacques Moreau), voire de mutation (Claude Vienney). Dans des banques coopératives ou mutualistes, les opérations bancaires peuvent poursuivre des finalités autres que celles qu’avaient fixées les fondateurs, et elles sont souvent plus compréhensibles à partir d'une approche en termes de croissance économique qu'en termes de services aux sociétaires. Dans l'agroalimentaire, des coopératives engagées dans la constitution d'un grand groupe sont fréquemment amenées à choisir la délocalisation plutôt que la territorialisation. René Mauget, pour le domaine agricole, et Jean-Noël Ory, Emmanuelle Gurtner et Mireille Jaeger, dans le secteur bancaire, ont décrit et analysé les concurrences d'intérêts qui naissaient dans les coopératives hybrides entre actionnaires et sociétaires.

De même que les coopératives et les mutuelles, des associations sont touchées par la confrontation directe au marché et peuvent, y compris dans le but de maintenir l'emploi, s'engager dans des démarches purement marchandes.

Ces évolutions sont plus souvent contraintes ou consenties que voulues: la proximité du marché ou de l'Etat brûle fréquemment les ailes du projet fondateur.

La banalisation des entreprises de l'ESS est aussi celle de leurs relations avec l'économie classique. 
Ainsi le 24 mars 2011, le Mouves et le Labo de l'économie sociale et solidaire se sont associés avec Entrepreneurs d'avenir (Generali) et Ashoka pour fonder l'Alliance pour entreprendre autrement. Jacques Huybrechts, fondateur du réseau Entrepreneurs d'avenir, considère que " l'Alliance pour entreprendre autrement vise à créer une dynamique de coopération entre plusieurs réseaux. [...] Nous souhaitons développer une identité partagée. Cela passera notamment par la signature commune d'une tribune à paraître dans la presse nationale ». Arnaud Mourot, directeur général d'Ashoka France, précise: "L'enjeu est de fédérer les initiatives disséminées, de les faire changer d'échelle et de créer des passerelles avec le monde des affaires."

Le développement d'une identité partagée et la création des passerelles avec le monde des affaires sont déjà bien engagés à travers les participations actives de leaders du Mouves dans Ashoka et dans le Boston Consulting Group.

L'interpénétration de l'ESS et de l'économie capitaliste est donc, sous des formes différentes, réelle aussi bien dans des associations les plus récentes et actives de la nouvelle ESS que dans certaines grandes organisations de l'ESS. Ajoutons immédiatement: de nombreux grands groupes de l'ESS ainsi que de nombreuses jeunes ou moins jeunes associations se tiennent à plus grande distance de l'économie dominante. Au contraire, ils revendiquent non seulement de porter une économie a-capitaliste, mais aussi un projet d'une société plus solidaire.

Plutôt qu'une opposition entre l'économie sociale historique et une nouvelle ESS, nous voyons ainsi se dessiner une distinction entre, d'une part, un pôle qui adosse sa dynamique économique sur celle de l'économie dominante, pôle qui réunit des groupes d'ESS, des groupes capitalistes et des entrepreneurs sociaux, et, d'autre part, un pôle qui fonde sa dynamique indépendamment de celle de l'économie dominante et qui réunit des groupes d'ESS, des fédérations et des petites associations, coopératives et entrepreneurs sociaux.

Quelles que soient les options de chacun - toutes sont évidemment respectables -, la question de la relation avec l'économie dominante, hier encore assez largement ignorée, est devenue centrale: de la réponse que l'ESS donnera à cette question dépend assez largement son projet d'avenir. La lecture des quatre cents cahiers d'espérance rédigés au cours des derniers mois donne la possibilité d'approfondir cette question cruciale... et sans doute de faciliter la traduction des cahiers en pratiques d'espérance.

\section{"Varia "}

Nos lecteurs trouveront dans ce numéro 321 une nouvelle rubrique. Répondant à un souhait exprimé de longue date par une partie de notre lectorat, la rubrique " Varia » réunit des études plus courtes et de lecture plus aisée - mais tout aussi rigoureuses - que celles qui font le corps de la Recma. 